\title{
Médiévales
}

Langues, Textes, Histoire

66 | printemps 2014

Harmonie Disharmonie

\section{Guillaume Crétin et la Déploration sur le trépas de Jean Ockeghem : les chœurs, les cœurs et la poésie} Guillaume Crétin and the Déploration sur le trépas de Jean Ockeghem: Choruses, Hearts and Poetry

\section{Thibaut Radomme}

\section{OpenEdition}

\section{Journals}

Édition électronique

URL : https://journals.openedition.org/medievales/7253

DOI : $10.4000 /$ medievales. 7253

ISSN : 1777-5892

Éditeur

Presses universitaires de Vincennes

Édition imprimée

Date de publication : 30 juin 2014

Pagination : 121-139

ISBN : 978-2-84292-405-8

ISSN : 0751-2708

\section{Référence électronique}

Thibaut Radomme, « Guillaume Crétin et la Déploration sur le trépas de Jean Ockeghem : les chœurs, les cœurs et la poésie », Médiévales [En ligne], 66 I printemps 2014, mis en ligne le 05 juillet 2016, consulté le 23 avril 2022. URL : http://journals.openedition.org/medievales/7253 ; DOI : https://doi.org/

$10.4000 /$ medievales.7253 


\section{Guillaume Crétin et la Déploration sur le trépas de Jean Ockeghem: les chœurs, les cœurs et la poésie}

L'histoire littéraire a conservé un souvenir modeste de Guillaume Crétin ${ }^{1}$. Né vers 1460 et mort le 30 novembre 1525 , il appartient à la seconde génération des Grands Rhétoriqueurs, nom sous lequel le $\mathrm{XIX}^{\mathrm{e}}$ siècle a rassemblé des poètes officiant à la cour des ducs de Bourgogne, de Bretagne et de Bourbon, ainsi qu'auprès du roi de France, durant la seconde moitié du $\mathrm{Xv}^{\mathrm{e}}$ et le premier tiers du $\mathrm{XvI}^{\mathrm{e}}$ siècle ${ }^{2}$. Guillaume Crétin, qui fut chanoine et trésorier de la Sainte-Chapelle de Vincennes puis chantre de la Sainte-Chapelle du Palais, à Paris, et aumônier ordinaire du roi François I ${ }^{\text {er }}$, a composé des Chroniques et des œuvres poétiques que Kathleen Chesney, dans son édition, propose de répartir en poésie religieuse, récréative (narrative ou épistolaire) et sérieuse ${ }^{3}$. Cette dernière catégorie compte des satires et cinq déplorations. C'est l'une de celles-ci, consacrée à Jean Ockeghem ( $c a$ 1420-6 février 1497), représentant célèbre du style polyphonique franco-flamand, que cet article entend étudier.

Écrite entre 1496 et 1499, la Deploration sur le trespas de feu Okergan $^{4}$ est un petit poème de quatre-cent-vingt décasyllabes, épousant

1. G. Tilander et F. Fery-Hue, «Guillaume Crétin», dans G. Hasenohr et M. Zink éd., Dictionnaire des lettres françaises. Le Moyen Âge, Paris, 1992, p. 614; J.-P. Bordier, «Guillaume Crétin», dans M. Simonin éd., Dictionnaire des lettres françaises. Le XVI siècle, Paris, 2001, p. 310-311.

2. À propos de ce courant poétique, voir par exemple Les Grands Rhétoriqueurs, Actes $d u \mathrm{~V}^{\mathrm{e}}$ Colloque International sur le Moyen Français, Milan, 1985; G. Di Stefano et R. M. BILder éd., La Grande Rhétorique. Hommage à Paul Zumthor, Le Moyen Français, 34 (1994); J. Koopmans éd., Rhetoric-Rhétoriqueurs-Rederijkers, Amsterdam, 1995. Sur l'origine de l'appellation Rhétoriqueurs, voir P. Zuмthor, Le Masque et la lumière. La poétique des Grands Rhétoriqueurs, Paris, 1978, p. 9.

3. Guillaume Crétin, Euvres poétiques, éd. K. Chesney, Paris, 1932. Toutes les citations du texte sont tirées de cette édition, où ce dernier occupe les pages 60-73.

4. Ibid., p. XXXIV. 
«la discrète et monotone fluidité du poème à rimes plates ${ }^{5}$ » qui sied bien à l'expression de la douleur. En voici un court résumé. La déploration commence par une introduction (v. 1-26), dans laquelle l'Auteur - ainsi qu'il se désigne lui-même -, épuisé par ses considérations sur l'universalité de la mort, accablé de fatigue, tombe dans un sommeil profond. Au cours de ce sommeil survient un songe (v. 27-40): l'Auteur se trouve «transporté devant le monument» (v. 28) mortuaire du compositeur. Après un temps d'oscillation entre fiction et réalité, il bascule pleinement dans sa rêverie (v. 41-240) et décrit la procession de Dame Musique et de ses gens devant le «sepulchre de marbre» (v. 56) d'Ockeghem: précédée des muses, la Musique enjoint aux personnages présents de chanter en mémoire du compositeur défunt. Ce sont alors Tubal, le roi David, Orphée et le centaure Chiron qui, chacun à son tour, chantent un rondeau: quatre rondeaux, de forme traditionnelle, attribués à ces quatre figures tutélaires de la mythologie musicale ${ }^{6}$, sont alors insérés dans la trame du poème. Dans la procession, on trouve encore la poétesse Sappho, Mercure, Pan et Arion (v. 185-208), puis un grand nombre de compositeurs illustres de la génération précédant celle d'Ockeghem, parmi lesquels Guillaume Dufay, Gilles Binchois ou John Dunstable, ainsi que quelques contemporains: ils sont «plus de vingt» (v. 210), note l'Auteur. Dame Musique fait ensuite signe à ce dernier, qui se tient appuyé contre un arbre à l'écart, et lui commande de mettre par écrit tout ce qu'il a vu et de transmettre aux générations futures

5. R. Deschaux, «Le Lai et la Complainte», dans Grundriss der romanischen Litteraturen des Mittelalters, vol. VIII/1, D. PoIRION éd., La Littérature française aux XIV et Xve siècles, Heidelberg, 1988, p. 81.

6. Si les personnages d'Orphée et du roi David ne posent pas question, il convient de s'attarder un instant sur Chiron: initié à la musique par Apollon, réputé pour sa grande co s'a (1' est une figure

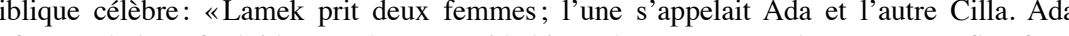
nfanta Yabal; ce fut lui le père de ceux qui habitent des tentes avec des troupeaux. Son frère s'appelait Youbal; ce fut lui le père de tous ceux qui jouent de la cithare et du chalumeau. Cilla, quant à elle, enfanta Toubal-Caïn qui aiguisait tout soc de bronze et de fer; la sœur de Toubal-Caïn était Naama.» (Gn 4, 19-22) Dans la Bible, Jubal est donc le père des musiciens, alors que Tubal est celui des forgerons. Pourtant, Isidore de Séville écrit dans ses Etymologiae, III, xv, 1: «Moyses dicit repertorem musicae artis fuisse Tubal, qui fuit de stirpe Cain ante diluuium» ( «Selon Moïse, l'inventeur de la musique est Tubal, de la race de Caïn, avant le déluge») (Isidorus Hisparensis, Etymologiae. III : De mathematica, éd. et trad. C. (c) Tubal t l'épisode de la forge dans la mythologie pythagoricienne d'autre part: «De saint Isidore de Séville (ca 559-636) à Praetorius (1571-1621), Jubal et Pythagore sont mentionnés ensemble comme inventores musicae. Isidore attribue à Jubal l'invention de la musique, et à Pythagore la découverte des principes à partir du son des marteaux d'un forgeron» (C. АвROMOnT, E. De Montalembert éd., Guide de la théorie de la musique, Paris, 2001, p. 373). Guillaume Crétin se fait l'écho de cette confusion: «Tubal le bon pere ancien, Qu'on dit et tient premier musicien Qui sur marteaux trouva sons et accordz» (v. 101-103). l'héritage musical d'Ockeghem. L'Auteur se réveille et commence à se lamenter sur son incapacité à rendre le génie du compositeur (v. 241-420):

il fait successivement appel aux poètes anciens (v. 253-264) puis aux poètes modernes (v. 265-312), prononce finalement l'éloge d'Ockeghem (v. 312 396) et exhorte ses contemporains au chant pour célébrer le musicien défunt (v. 397-420).

\section{Contexte et problématisation}

S'il est une notion riche et complexe mise en scène dans le poème de Guillaume Crétin, c'est bien celle de l'harmonie - ainsi que son antithèse la disharmonie. À ce sujet, il n'est sans doute pas nécessaire de rappeler combien le principe pythagorico-platonicien du nombre et des proportions a structuré la pensée médiévale de la musique ${ }^{7}$. L'idée selon laquelle la musique serait le miroir de l'harmonie universelle est en effet transmise de manière ininterrompue de l'Antiquité au Moyen Âge, par l'intermédiaire de Plotin (Ennéades), Boèce (De musica), Cassiodore (Institutiones) et Isidore de Séville (Etymologiae), en passant par Augustin, qui écrit dans le De ordine (II, 5,14$)$ que, «dans la musique, dans la géométrie, dans les mouvements des astres, dans les nécessités des nombres, l'ordre domine [ordo ita dominatur] de telle sorte que, si l'on voulait en voir quasiment la source ou le voir en son sanctuaire, c'est là qu'on le trouverait ou par là qu'on y serait conduit sans erreur aucune ${ }^{8} »$. Ainsi, l'univers est supposé avoir été ordonné par Dieu et l'homme peut accéder à la connaissance de cet ordre cosmique par la pratique des arts du quadrivium qui, tels les degrés d'une échelle, conduisent l'âme humaine à la contemplation de la perfection divine $^{9}$. Cette conviction selon laquelle l'étude de la musique ouvre l'accès

7. Ce survol extrêmement hâtif de l'histoire de la pensée musicale au Moyen Âge est un résumé de l'article de M. GARDA, «Esthétique. Petite histoire des conceptions du beau musical», dans J.-J. NATTIEZ éd., Musiques. Une Encyclopédie pour le XXI e siècle, t. II, Les Savoirs musicaux, Arles, 2004, p. 649-671.

8. «[...] iam in musica, in geometrica, in astrorum motibus, in numerorum necessitatibu ordo ita dominatur, ut, si quis quasi eius fontem atque ipsum penetrale uidere desideret, aut in his inueniat aut per haec eo sine ullo errore ducatur» (SAncti Aureli Augustini,Contra academicos, De beat academicos, De beata vita,

9. Dans les Confessions, X, 33, Augustin souligne le caractère ambivalent du chant liturgique, notant qu'il incline à la piété les âmes des fidèles, tout en confessant que les «voluptés des oreilles» ( «uoluptates aurium») risquent de les distraire de la prière. Il conclut «ita fluctuo inter periculum uoluptatis et experimentum salubritatis» («ainsi j’hésite entre le danger du plaisir et l'expérience de l'utilité»), avant de se prononcer finalement plutôt en faveur («magisque adducor») du maintien du chant dans l'Église (SANCTI AureLII AugustinI, Confessionum libri XIII, éd. L. VerheIJen, CCSL, 27, Turnhout, 1981). Cette hésitation augustinienne sera le terreau des condamnations que l'Église prononcera à l'encontre de 
à des réalités transcendantes explique que la notion d'harmonie - reflet de l'harmonie universelle - soit au cœur de la réflexion musicale médiévale; elle permet aussi de comprendre la distinction marquée par le Moyen Âge entre le musicus et le cantor, qui se traduit par la valorisation du premier, théoricien, et la dépréciation du second, praticien. Ce n'est qu'au XII siècle qu'émerge, au cœur des premiers véritables traités de composition musicale, la problématique du beau comme prescription esthétique. Le principe grec de la proportion comme fondement éthique d'une relation musicale au divin n'est dès lors plus l'objet exclusif de l'attention des penseurs, et l'approche matérielle du cantor, soucieuse de la douceur des sons et du plaisir esthétique de l'auditeur, se trouve valorisée.

Ce nouveau paradigme ouvre la voie à un champ d'investigation inédit, exploré d'abord par Marchettus de Padoue ( ca 1270-1335) dans son Lucidarium : la relation des dissonances et des consonances en musique. Il était nécessaire en effet que la musique sonore se détachât de son avatar intellectualisé, reflet de l'harmonie forcément parfaite de la Création divine, pour qu'émergeât la possibilité d'une conception contrastée de la musique, entre harmonie et disharmonie: «Une succession correcte d'intervalles consonants et dissonants constitue la substance de la composition polyphonique : la beauté n'est donc pas uniquement le reflet du beau intelligible que l'intellect saisit dans l'œuvre musicale comme dans le cosmos ou l'harmonie du corps humain; elle est dotée du caractère propre à la musique sonore ${ }^{10}$.» Au milieu du $\mathrm{XVI}^{\mathrm{e}}$ siècle, le célèbre théoricien de la musique Gioseffo Zarlino écrira que le contrepoint est rendu «plus joyeux et plus beau ${ }^{11} »$ par les dissonances accidentelles dont il est émaillé. Il ajoute: «[...] l'on ne doit pas pour autant comprendre qu'il faut les placer dans les Contrepoints, ou les Compositions, comme cela se pratique, sans aucune règle et sans aucun ordre: car cela provoquerait de la confusion; il faut au contraire s'obliger à les placer avec ordre et selon des règles, afin que le tout

la musique et de ses beautés sensibles de façon très remarquable en l'année 813: quatre des cinq conciles qui se tiennent alors légiferent, en des termes très semblables, contre «the performances of matter not fit for clerical ears and eyes, including music » (M. RichteR, The Oral Tradition in the Early Middle Ages, Turnhout, 1994 (Typologie des Sources du Moyen Âge Occidental, 71), p. $51 ; c f$.les conciles de Mayence (canon XIV), Chalon-sur-Saône (canon IX), Reims (canon XVII) et Tours (canon VII) : Concilia aevi Karolini, éd. A. Werminghoff, MGH Conc 2 1, Hanovre 1906). L'Église répétera cet interdit, à travers la bulle papale de Jean XXII . 1322 contre les excès de l'A 's nova, ou encore lors de la vingt-deuxiène session Jen XXII en

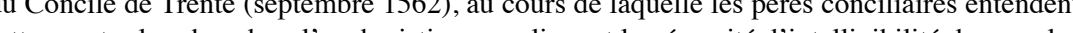
utter contre les abus dans l'eucharistie en soulignant la nécessité d'intelligibilité des paroles des chants liturgiques et en rejetant les matériaux musicaux d'origine profane. Bien entendu, c'est à chaque fois la crainte des séductions de la musique, de son ravissement des sens et du trouble dans lequel elle jette l'esprit qui nourrit ces anathèmes divers.

10. M. GARDA, «Esthétique...», p. 657.

11. Gioseffo Zarlino, Le Istituzioni armoniche, Venise, 1558 (rééd. de l'édition de 1573: Ridgewood, 1966),p. 157 (cité par M. GARDA, «Esthétique...», p. 658). sonne bien ${ }^{12}$.» Consonance, dissonance; harmonie, disharmonie: le débat au croisement de la spéculation et de la pratique musicale, est complexe et sous-tend l'apprivoisement de la musique polyphonique par la civilisation de l'Occident médiéval. C'est, sans aucun doute, dans cet arrière-fon culturel qu'il convient de placer le poème de Guillaume Crétin, c'est à la lumière de ces interrogations, aussi bien éthiques qu'esthétiques, qu'il est nécessaire de le lire pour en éclairer les enjeux intellectuels.

Sur le plan littéraire, la Déploration est une petite pièce assez intéressante: elle exploite en effet quelques-uns des phénomènes poétiques en grande vogue à la fin du Moyen Âge. Elle appartient d'abord au genre de la complainte, «une forme littéraire fréquente dès le $\mathrm{xI}^{\mathrm{e}}$ siècle ${ }^{13}$ », «issue peut-être du planctus de la chanson de geste ou du planh des troubadours, ou encore simple variété de sirventès, inspiré de la mort d'un grand personnage ou d'un être cher ${ }^{14}$ ». S'il nous reste des complaintes des XII et XIII siècles, souvent accompagnées de musique, d'abord en langue d'oc puis en langue d'oïl, c'est en moyen français qu'a lieu la grande floraison du genre, caractérisé par une importante variété formelle, certains auteurs faisant même le choix de la prose ${ }^{15}$. Dès lors, le seul trait véritablement commun aux avatars divers du genre est son contenu thématique: «L'émotion douloureuse qui suscite la complainte peut avoir des causes multiples [...]. Mais les trois désarrois les plus fréquemment repérables à l'origine de ce genre de poème proviennent des méfaits de la politique et de la guerre, de la déception amoureuse, de la mort d'un être cher, d'un personnage important, voire d'un animal domestique ${ }^{16}$.» Plus spécifiquement, au sein des textes chantant ce troisième type de désarroi, la complainte inspirée par la mort d'un artiste n'est pas rare, de la Complainte de la mort de Jacques Milet de Simon Gréban (1466) à l'Épitaphe de Jean Molinet pour le peintre Simon Marmion (1489), en passant par la Complainte de la mort de maistre George Chastellain tresclair orateur de Jean Robertet (1476): «faisan appel à la mythologie et aux écrivains antiques, mettant en scène Nature, Art et Imitation, il vante les talents du défunt, auxquels il oppose sa propre médiocrité ${ }^{17} . »$ Cette description n'est évidemment pas sans rappeler la Déploration de Guillaume Crétin, lequel inscrit donc son poème dans le réseau de références et de significations d'un genre semblant être devenu topique au dernier siècle du Moyen Âge.

12. Ibid., p. 172

13. Jean Robertet, Euvres, éd. M. Zsuppan, Genève, 1970, p. 52 (cité par R. Deschaux, «Le Lai et la Complainte...», p. 71).

14. R. Deschaux, «Le Lai et la Complainte...», p. 71

15. Ibid., p. 71-72.

16. Ibid., p. 81 .

17. Ibid p. 82 
Par ailleurs, la Déploration met en scène un songe allégorique (ou songe autobiographique), «un mode d'écriture majeur au Moyen Âge et, sans aucun doute, le plus significatif à partir de la fin du XIII ${ }^{\mathrm{e}}$ siècle $^{18}$ ». S'appuyant sur la définition a minima du songe autobiographique («Je raconte que je a songé ou vu des puissances surnaturelles $\left.{ }^{19} »\right)$, Pierre-Yves Badel en établit une typologie très précise à partir des formules d'introduction et de conclusion qui en délimitent le cadre ainsi que du rôle assigné au rêveur à l'intérieur du songe. Il ajoute que le songe se caractérise par «la généralité du message et l'unicité de l'expérience», puisqu'il est à la fois «aventure singulière, unique, qui tend à se faire passer pour un fragment de la vie individuelle du narrateur » et «enseignement qui vaut pour tous »d'un initié racontant la vision surnaturelle qu'il a eue. Dès lors, «la fiction $d u$ songe rend la parole d'un poète plus solennelle $»^{20}$. Je tenterai d'interroger la Déploration à la lumière de ceci, et montrerai comment l'Auteur exploite cette solennité nouvelle que le récit de son rêve lui confère.

Les quatre rondeaux de la Déploration relèvent du procédé de l'insertion lyrique, à propos duquel Jacqueline Cerquiglini-Toulet note qu'en mêlant narration et chant, récit et lyrisme, il matérialise l'«interrogation sur la forme qui hante les $\mathrm{XIV}^{\mathrm{e}}$ et $\mathrm{XV}^{\mathrm{e}}$ siècles ${ }^{21} »$. À son tour, Jacqueline Cerquiglini-Toulet propose une typologie de l'insertion lyrique, distinguant le collage du montage du point de vue de la mise en rapport des formes, de l'énonciation et du but de l'insertion ${ }^{22}$. Il serait pertinent d'appliquer ces critères de classification à la Déploration; l'on constaterait ainsi que l'insertion qui y est pratiquée est d'un genre hybride (situation que Jacqueline Cerquiglini-Toulet a du reste prévue, en établissant «une série de critères qui, diversement combinés, engendrent une matrice de possible $\left.{ }^{23} »\right)$. On peut cependant résumer en disant que les rondeaux de la Déploration relèvent globalement du premier type d'insertion lyrique, puisqu'ils «fonctionne[nt] à la manière d'une citation, confirmant [...] le propos de la narration en vers» et qu'ils affirment la non-coïncidence

18. P.-Y. BADEL, Le Roman de la Rose au XIV siècle. Étude de la réception de l'œuvre, Genève, 1980, p. 332. Sur ce sujet, voir également P.-Y. BADEL, «Le poème allégorique», dans Grundriss der Romanischen Litteraturen..., vol. vIII/1, D. Poirion éd., La Littérature française aux $\mathrm{XIV}^{e}$ et $\mathrm{xV}^{e}$ siècles..., p.139-160; A. STRUBEL, « Grant senefiance $a$ » : allégorie et littérature au Moyen Âge, Paris, 2002; H. R. JAUss et U. EBEL, «Entstehung und Strukturwandel der allegorischen Dichtung", dans Grundriss der Romanischen Litteraturen .. vol. vil, der allegorischen Dichtung», dans Grundriss der Romanischen Litteraturen..., vol. vI/1,

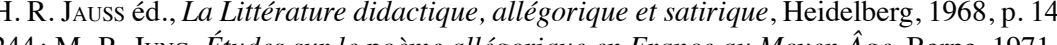

44; M.-R. Jung, Études sur le poème allégorique en France au Moyen Âge, Berne, 1971.

19. P.-Y. BADEL, Le Roman de la Rose $a u$ XIVe siècle..., p. 340.

20. Ibid. p. 348 (souligné par l'auteur).

21. J. Cerquiglins, «Un engin si soutil». Guillaume de Machaut et l'écriture au XIV siècle, Paris, 1985, p. 23.

22. Ibid., p. 24-28.

23. Ibid., p. 29 du je lyrique avec le je de la narration, bien qu'ils «figurent avec le titre de leur forme, titre qui les isole et les met en valeur ${ }^{24} \gg$ : cette exposition est caractéristique du montage, par opposition à l'assimilation propre au collage. Quoi qu'il en soit de cette question, l'essentiel est de s'interroge sur la fonction de l'insertion lyrique; j'y reviendrai au cours de cette étude.

Enfin, le quatrième phénomène poétique est la mobilisation d'un personnel littéraire nombreux, issu de traditions diverses, dans le songe aussi bien que dans le poème-cadre. Outre le fait que de telles galeries de personnages soient, comme on l'a vu, rendues nécessaires par la logique même, encomiastique ou visionnaire, de la complainte et du songe allégorique, leur foisonnement semble répondre au goût du siècle. Robert Deschaux note ainsi que, «[c]omme la plupart des formes poétiques de l'époque», la complainte fait «une place grandissante à la mythologie gréco-latine, le personnage mythologique n'excluant du poème ni la personnification allégorique, ni le personnage biblique ou historique $\gg^{25}$.

À la lumière de ces prolégomènes, la question que cet article veut poser est double. Il s'agit dans un premier temps de montrer comment la Déploration confronte les représentations de l'harmonie des chœurs et de la disharmonie des cœurs, ce qui soulève le paradoxe du chant-plainte. Dans un second temps, j'analyserai le topos d'humilité tel qu'il est mis en scène et problématisé par le poète, en l'articulant avec les catégories génériques et formelles que j'ai dégagées ci-dessus.

\section{Harmonie et disharmonie}

La description de la disharmonie des cœurs déchirés par la tristesse est l'occasion d'une thématisation de la douleur au moyen d'un vocabulaire très expressif (je souligne à chaque fois)

Musiciens se doibvent huy contraindre,

Et en grandz pleurs leurs cueurs baigner et taindre,

En le voyant ainsi mort allité (v. 116-118)

Vos cueurs debvez en couroux tourmenter,

Et de regretz vos ennuys augmenter,

Car huy perdez la fleur de voz amys (v. 154-156)

Les cœurs, tourmentés par le chagrin, baignés de pleurs, sont le siège des sombres sentiments de l'assistance. Tout au contraire, les chants son

24. Ibid., p. 24 et 27.

25. R. DesCHaUX, «Le Lai et la Complainte...», p. 84 
harmonieux : «Puis en l'instant la compaignie assemble, Et instrumentz fait accorder ensemble» (v. 71-72). L'Auteur met en scène une opposition entre Sappho et Atropos, l'une des trois Moires grecques, divinités du Destin auxquelles correspondent les Parques romaines ${ }^{26}$. L'harmonie du chant de l'amoureuse de Pan, «plain d'argus », c'est-à-dire plein de subtilité (DMF 2012, s.v. argu), contredit la disharmonie suprême de la mort:

Dame Sapho, de Pan belle amoureuse,

Contre Atropos austere et rigoreuse

Feit et chanta ung dictié plain d'argus. (v. 185-187)

Enfin, ces musiciens qui célèbrent la mémoire d'Ockeghem sont représentés avec une assemblée nombreuse d'auditeurs se délectant de l'harmonie de leur musique:

Maint homme fut aupres d'eulx escoutant

Car bon faisoit ouyr telle armonye

Aussi estoit la bende bien fournye. (v. 214-216)

Cependant, la frontière qui semblait bien dessinée entre l'harmonie des chœurs et la disharmonie des cœurs se trouve soudain brouillée. Les chants sont gagnés par le trouble des pleurs, dans le rondeau de David:

En chant de pleur doibt bien psalmodier

Tout bon esprit, et bien estudier

A lamenter ce Tresorier notable ${ }^{27}$ (v. 128-130)

Plus loin, c'est le centaure Chiron qui, d'une voix tremblante et tout en pleurant, se met à chanter son rondeau. Sa voix sonne «un peu cas»: cas est un adjectif signifiant, pour une personne, «faible, fragile, débile, décrépit, brisé » et, par analogie, pour une émission sonore, «faible, bas» (DMF 2012, s.v.cas). Le Dictionnaire du Moyen Français relève également l'expression sonner cas, «émettre un son sourd, faible», voire «sonner faux».

Aux assistens fort contristez du cas,

De voix tremblant, resonnant un peu cas,

26. Il avait déjà été fait mention d'Atropos au début du poème: «[...] la fiere Atropos, Qui frappe, fiert et rue a tout propos Sur papes, roys, empereurs, ducs et contes; [...] elle mect en ses comptes Tant clercz que layz, tant nobles que villains, Tant grans prelatz que paovres chapellains » (v. 5-10).

27. Ockeghem fut le trésorier de l'abbaye Saint-Martin de Tours.
Piteusement la matiere poursuyt,

Et en plorant dit le mot qui s'ensuyt. [Rondeau de Chiron] (v. 166-169)

Le sens «sonner faux» paraît pertinent dans ce contexte: il n'y a rien d'étonnant, en effet, à ce qu'un chanteur qui pleure et dont la voix tremble se mette à chanter un peu faux. Voilà donc une fissure dans la belle harmonie du chant telle qu'elle avait été mise en scène par l'Auteur: les chœurs sont gagnés également par la disharmonie des cœurs. Le poète parle encore de «chantz piteux» (v. 53) et de «piteux son» (v. 100). Ainsi, quelques mentions dysphoriques essaiment dans la Déploration et annoncent la conjonction des pleurs et des chants, avant la confusion complète en fin de poème. Le statut des chants semble donc plus équivoque qu'à première vue : ils sont doux et harmonieux certes, mais en même temps pitoyables et baignés de pleurs. L'opposition harmonie/disharmonie ne sert plus uniquement de ligne de démarcation entre les chœurs et les cœurs mais elle est présente jusque dans le chœur.

Au-delà de la question de l'harmonie ou la disharmonie des chœurs, le poème procède à un rejet des chants joyeux : la tristesse des cœurs doit gagner les chants, parce que la gaieté ne saurait convenir à la situation funèbre.

De chantz plaisans ne fault plus guermenter,

Mais en douleurs vous experimenter,

Ainsi que gens de tous plaisirs remis (v. 158-160)

Ne parlez plus de joyeux chantz ne ris,

Mais composez ung Ne recorderis,

Pour lamenter nostre maistre et bon pere. (v. 399-401)

Ce refus de la joie musicale entre en contradiction nette avec les vers célèbres du Prologue de Guillaume de Machaut, qui affirment la contiguïté inaliénable de la musique et de la gaieté ${ }^{28}$ :

Et musique est une science

Qui vuet qu'on rie et chante et dance.

Cure n'a de merencolie,

Ne d'homme qui merencolie

A chose qui ne puet valoir,

Eins met tels gens en nonchaloir.

Partout ou elle est, joie y porte

Les desconfortez reconforte,

28. Gulllaume de Machaut, Eeuvres, éd. E. Hoepffner, Paris, 1908, t. I, p. 9, v. 85-94. 
Et nès seulement de l'oïr

Fait elle les gens resjoïr.

Comme le suggère Agathe Sultan, «[1]'art musical, indissociablement lié à la réjouissance, pourrait donc affronter un paradoxe dès lors qu'il doit affronter la mort ${ }^{29} \gg$. En effet, comment résoudre l'aporie résultant de l'injonction à la tristesse dans le chant faite par le poème et de l'essence joyeuse de la musique telle qu'elle est affirmée par Guillaume de Machaut? Le poète propose de résoudre ce paradoxe et de réconcilier les contraires en réclamant un chant, certes, mais un chant très sobre et en exigeant que l'harmonie de la musique soit gagnée par la disharmonie des pleurs:

Enffans du cueur, ne faictes plus leçons

De fleuretiz, mais note contre note

Sur Requiem en doulcettes façons ;

Puis accordez voz chantz et piteux sons,

Sans ce que aulcun rien y adjouste ne oste (v. 413-417)

L'Auteur ordonne d'abandonner les «fleuretiz», ces «fioriture[s], ornement[s] que les chantres improvisent sur le plain-chant» (DMF 2012, s.v. fleuretis ; FEW III, 630a, s.v. flos) : le chant doit être fait note contre note. En outre, les chants et les sons doivent être «piteux». Ainsi, la double opposition de la joie et de la plainte, d'une part, de l'harmonie et de la disharmonie, d'autre part, est neutralisée : plainte et chant, cœurs et chœurs se confondent dans la déploration du disparu.

Gens du clergé et college notable,

Chant lamentable en cueur et en chapitre

Faire debvez pour cest homme louable (v. 349-351)

Cette confusion du cœur et du chœur exigée par le poète me semble clairement soulignée par l'expression «en cueur et en chapitre»: n'y a-t-il pas là un jeu graphique volontaire destiné à marquer cet embrouillement jusque dans la langue? En effet, le mot attendu dans cette expression est chœur (et non cœur), qu'il s'agisse de chanter en chœur ou dans le chœur. Certes, les variantes graphiques chœur et cœur se confondent en moyen français pour désigner le chœur ( $c f$. DMF 2012, s.v. chœur) ${ }^{30}$; il convient

29. A. Sultan, «Tombeaux de musiciens à la fin du Moyen Âge», dans E. Doudet éd., La Mort écrite. Rites et rhétoriques du trépas au Moyen Âge, Paris, 2005, p. 157.

30. L'alternance orthographique persiste aux $\mathrm{XVI}^{\mathrm{e}}$ et $\mathrm{XVII}^{\mathrm{e}}$ siècles dans les dictionnaires de R. Estienne (1549), J. ThierRy (1564) et J. Nicot (1606), quoiqu'Estienne connaisse l'étymologie véritable du mot: «Semble qu'il uienne de chorus, et qu'il fault escrire chøur», ainsi que Thierry et Nicot qui font la même remarque. Il faut attendre la première édition donc d'être prudent à ce sujet. Mais le syntagme «enffans $d u$ cueur» (v. 413) n'est-il pas un autre indice de ce jeu, alors que la forme attendue est enfant de c(h)œur? On sait les Grands Rhétoriqueurs friands de ces jeux formels très subtils ${ }^{31}$. Il me semble donc bien, pour toutes ces raisons et malgré les réserves que j'ai émises, que le poète s'est plu à traduire ici la confusion des cœurs et des chœurs sur le plan du signifiant linguistique.

\section{Musique et poésie}

Afin d'analyser pertinemment le poème, il convient de noter l'injonction du geste littéraire qui est faite à l'Auteur par Dame Musique. Celle-ci lui commande en effet, comme je l'ai souligné, de raconter ce qu'il a vu et de transmettre à la postérité la doctrine et les enseignements d'Ockeghem:

Musique lors, la dame tresdolente [...]

Me commanda estre prest et pourveu

D'enregistrer tout ce que j'avoye veu.

Oultre me dit et chargea par expres

De publier et dire loing et pres

Aux chantres tous sa doctrine ensuyvans (v. 224-233)

À la suite de cette injonction, l'Auteur se réveille et se met aussitôt à se lamenter: il est un mauvais poète. Dès lors, comment pourrait-il, avec ses moyens poétiques limités, mettre en mots la beauté des chants funèbres qu'il a entendus, et même rendre hommage et justice au génie d'Ockeghem? «La raison veult que memoire en façon, Mais ad ce suis trop inutile ouvrier.» (v. 275-276) En appelant aux grands poètes de son temps, il affirme qu'ils pourraient bien mieux que lui remplir la tâche qui lui a été confiée: «Plustost de luy deussiez faire cantique, Que moy qui suis en elegance etique » (v. 292-293). Poète blâmé ou méprisé, il ne reçoit en effet de ses contemporains que ce que ses écrits, frustes et laborieux, méritent: «a bon droict on me blasme ou mesprise Pour mon escript rural et mecanicque» (v. 296-297). L'Auteur est d'autant plus impuissant que le génie d'Ockeghem est magistral et que la représentation qu'en fait Guillaume Crétin est hiératique: les appellations du compositeur méritent

du Dictionnaire de l'Académie française (1694) pour que l'orthographe étymologique soit imposée, et que les deux homophones soient distingués de façon prescriptive ( $c f$. «chœur n. m. "chorale"», dans N. CATACH éd., Dictionnaire historique de l'orthographe française, Paris, 1995, p. 235b; Estienne est cité d' après cet ouvrage).

31. $C f$. par exemple J. Cerquiglini-Toulet, «L'éclat de la langue. Éléments d'une esthétique des Grands Rhétoriqueurs», dans Grands Rhétoriqueurs, Cahiers V.-L. Saulnier , XIV (1997), p. 75-82 
à ce titre quelque attention. En effet, si Guillaume Crétin met d'abord en scène une proximité forte et un rapport assez intime entre l'artiste et lui-même, l'appelant le «bon seigneur que franchement amoye» (v. 29), il le nomme plus loin le «filz» de Dame Musique (v. 62 et 99), créant, par l'intermédiaire de cette prosopopée, une distanciation qui concourt à la consécration d'Ockeghem. L'attribution d'une stature allégorique au musicien souligne en effet, par contraste, les propres limites du poète. Enfin, Guillaume Crétin écrit: «ce vaillant docteur» (v. 312) et «Docteur le puis nommer en la science» (v. 313). Il introduit ainsi une dimension sociale dans son éloge, sur le plan à la fois scientifique et institutionnel, puisqu'en recevant le titre de docteur, Ockeghem est considéré comme un savant: la musique est une science appartenant au quadrivium, au même titre que l'arithmétique, la géométrie ou l'astronomie. Guillaume Crétin valorise en outre la musique d'Ockeghem, évoquant «ses doulx chantz angelicques» (v. 390), en référence au topos de la musique et du chant comme attributs des anges, par opposition aux cris des diables. Bref, l'Auteur n'est pas à la hauteur:

Je suis perplex; en l'affaire qu'ay pris

Besoing me fust que aultre acteur mieulx apris

Vint a present mon sens ressusciter. (v. 250-252)

Ce topos d'humilité, tout conventionnel qu'il soit, est l'occasion d'une intéressante distinction entre la musique et la poésie. Alors que les musiciens chantent dans la douleur, malgré la douleur - le songe l'a assez montré -, Guillaume Crétin s'avoue incapable d'écrire:

Comment pourray sans me necessiter

En ce papier coucher ditz ne escriptz?

Veu que ne puis cueur ne bouche inciter,

Langue ne voix esmouvoir, n'exciter

A prononcer fors pleurs, plainctes et criz. (v. 242-246)

Il est impuissant à articuler quoi que ce soit sauf un pleur, un cri, c'est-à-dire l'antithèse de la parole poétique: à la langue harmonieuse, littérarisée, que suppose cette dernière, l'Auteur oppose la sauvagerie, l'inarticulation brute du cri. L'opposition harmonie/disharmonie se trouve donc transposée dans l'opposition musique/poésie, consacrant de ce fait la supériorité de la musique sur la poésie, puisque celle-là est apte, à la différence de celle-ci, à l'expression harmonieuse même dans la douleur. Ceci est à replacer dans le contexte de l'histoire littéraire et de l'histoire de la musique. C'est en effet au $\mathrm{Xv}^{\mathrm{e}}$ siècle que la séparation de la poésie et de la musique est consommée. En 1392, Eustache Deschamps livre son Art de dictier et de fere chançons, où il différencie la musique naturelle (la poésie) de la musique artificielle (la musique proprement dite) : «Et est a sçavoir que nous avons deux musiques, dont l'une est artificiele et l'autre est naturele ${ }^{32}$.» Eustache Deschamps constate d'ailleurs que les poètes ne connaissent pa la musique (à la différence de Guillaume de Machaut, figure emblématique du poète-musicien) : «Et ja soit ce [...] que les faiseurs d'icelle ne saichent pas communement la musique artificiele ne donner chant par art de notes a ce qu'ilz font, toutesvoies est appellée musique ceste science naturele ${ }^{33}$.» Le traité d'Eustache Deschamps débute donc par «une réflexion théorique sur la place de la poésie au sein des arts du trivium et du quadrivium ${ }^{34}{ }$. $_{\text {. }}$ «Deschamps ne situe pas la poésie du côté de la rhétorique et des arts du verbe, mais du côté de la musique ${ }^{35}{ }^{\prime}$ parce qu'elle est avant tout musique de la langue. À ses yeux en effet, «la poésie utilise secrètement les autres arts, elle est tout ensemble proportion et construction, nombre et mesure, discours et musique. En elle doit s'incarner la musicalité particulière de la voix et de la langue ${ }^{36} \gg$. Elle opère, pour ainsi dire, la synthèse des sept arts libéraux.

On quitte donc le XIV siècle de Machaut pour entrer dans l'ère du divorce: à mesure que la complexité de la polyphonie croît, le texte chanté devient de moins en moins compréhensible et passe au second plan; chant et poésie se séparent, et les Grands Rhétoriqueurs, à l'occasion de cette scission, revalorisent la poésie et lui confèrent de la musicalité par un soin tout particulier pour la forme et par des jeux linguistiques auxquels ils s'adonnent constamment ${ }^{37}$.

À la fin $\mathrm{du} \mathrm{XVI}^{\mathrm{e}}$ siècle, les compositeurs et théoriciens de l'entourage du comte Bardi, autour de la Camerata fiorentina, opéreront finalement un retour au texte: faisant la critique de la musique polyphonique, qui empêche l'intelligibilité du texte, et du contrepoint abusif, qui gêne la communication de l'affetto (l'émotion), ils promouvront le stile recitativo, mi-chanté mi-parlé, inspiré de la musique grecque antique ${ }^{38}$. C'est alors le développement de la monodie accompagnée et la naissance de l'opéra, qui consacre la réconciliation du texte et de la musique au tout début du $\mathrm{XVII}^{\mathrm{e}}$ siècle. On constatera donc que la distinction entre poésie et musique,

32. M. GaLly éd., Oc, oül, si. Les langues de la poésie entre grammaire et musique, Paris, 2010, p. 222.

33. Ibid., p. 224-226

34. Ibid., p. 212

35. Ibid.

36. Ibid., p. 213

37. Cf. J. Cerquiglini-Toulet, «L'éclat de la langue....».

38. C. Авromont et E. de Montalembert éd., Guide de la théorie de la musique...., p. 413 («Galilei, Vincenzo»), p. 416 («Bardi, Giovanni de») et p. 421 («Monteverdi, Claudio»). 
telle qu'elle est thématisée dans la Déploration, est tout à fait typique de l'époque de Guillaume Crétin.

Après avoir été un panthéon des grands musiciens dans le songe, le poème se fait le prétexte d'une litanie des grands poètes. En effet, puisqu'il s'affirme incapable de célébrer l'art d'Ockeghem, Guillaume Crétin appelle à son secours ses illustres prédécesseurs et ses contemporains célèbres. Il s'agit d'abord des grands poètes antiques:

Que n'euz je lors l'eloquence de Tulle,

Ou de Virgile, ou ceulx qu'on intitulle

Grands orateurs et poetes laurez?

Boece ou est il, qui ne me congratulle?

Ou est Properce et Tiburce ou Catulle,

Pour recueillir tous leurs escriptz dorez? (v. 253-258)

Ces références à l'Antiquité, et surtout ce souhait d'écrire à la façon des grands littérateurs latins, sont évidemment typiques, pour la littérature vernaculaire, du pré-humanisme des $\mathrm{XIV}^{\mathrm{e}} \mathrm{et}_{\mathrm{XV}} \mathrm{e}^{\mathrm{e}}$ siècles ${ }^{39}$. Le topos d'humilité s'inscrit dès lors dans un contexte historique et idéologique précis, qui se cristallise autour de l'espoir de retrouver l'éloquence antique par l'imitation, en particulier celle de Cicéron dès le XIV ${ }^{\mathrm{e}}$ siècle en Italie, que le mouvement du cicéronianisme affirmera au $\mathrm{XVI}^{\mathrm{e}}$ siècle comme le plus grand de tous les orateurs. Dès le début du Xve siècle, Alain Chartier (1385-1449), dont les Grands Rhétoriqueurs «se réclament volontiers ${ }^{40}$ », se qualifiait, dans le Quadrilogue invectif, de «lointaing immitateur des orateurs ${ }^{41} »$. Après avoir invoqué les poètes antiques, l'Auteur nomme ses prédécesseurs sur le mode nostalgique de l'ubi sunt:

Hé ! Chastelain et Maistre Alain Chartier

Ou estes vous? Il me fust bien mestier

Avoir de vous quelque bonne leçon;

Simon, Greban qui fustes du mestier,

Que n'avez vous laissé pour heritier

Ung Meschinot, ung Milet, ung Nesson,

Pour hault louer le melodieux son,

La voix, le chant et subtile façon

De ce vaillant renommé Tresorier (v. 265-273)

39. M. ZINK, Littérature française du Moyen Âge (Paris, 1992), Paris, 2004, p. 321-322 ( «Du clerc à l'humaniste»).

40. Ibid., p. 297

41. Alain Chartier, Le Quadrilogue invectif, éd. F. Bouchet, Paris, 2011, p. 3.
Cet appel en deux temps fait aux poètes pour célébrer le génie d'Ockeghem a deux effets: d'abord il légitime la poésie par rapport à la musique ensuite il légitime les poètes modernes par rapport à leurs pairs antiques (puisque les uns sont placés sur le même pied que les autres). L'Auteu semble ainsi hésiter entre ce que Curtius appelle la «modestie affectée ${ }^{42}$ » et les «topoi de l'ineffable», à propos desquels il note: «Au Moyen Âge on accumule les noms d'écrivains célèbres qui ne seraient pas à la hauteur de la tâche ${ }^{43}$ », à savoir ici la déploration d'un grand personnage. Guillaume Crétin détourne donc, en la renversant, cette ficelle rhétorique: il accumule les noms d'écrivains qui, précisément, seraient à la hauteur de la tâche, afin de souligner sa propre inaptitude et donc, en conséquence, la grandeur de Jean Ockeghem. Il valorise en outre les poètes qu'il invoque, quoiqu'il s'en défende: «En ce ne vueil vous estre adulateur» (v. 304). Guillaume Crétin fait donc preuve d'une grande finesse dans la façon dont il joue et se joue de la topique poétique.

\section{Prince des poètes}

En guise de conclusion, je voudrais poser la question suivante: cette Déploration ne serait-elle pas le lieu d'une auto-légitimation du poète, bien que celui-ci ait affirmé être incapable d'écrire et qu'il ait reconnu, pour cette raison, la supériorité de la musique sur la poésie ? En effet, il convient d'abord de s'attarder sur le paradoxe qui sous-tend le topos d'humilité, ici comme à chaque fois qu'un écrivain l'utilise: alors qu'il ne cesse de dire qu'un meilleur poète devrait écrire cet hommage, l'Auteur est précisément en train de l'écrire; quant à raconter ce qu'il a vu, c'est même chose faite puisqu'au moment où il s'accable, le poète a déjà rapporté son rêve. Le topos d'humilité affirme ainsi le poète incapable de ce qu'il est précisémen occupé à faire. Plus la tâche accomplie est ardue, plus le topos d'humilité est donc paradoxalement orgueilleux. Ici, la tâche est très difficile étant donné qu'il s'agit de rendre en mots le génie d'Ockeghem et l'harmonie des chants du songe. En outre, Guillaume Crétin se compare implicitement aux grands poètes qu'il a invoqués puisqu'il a mené à bien l'ouvrage qu'il leur demandait d'accomplir à sa place. Le topos d'humilité produit donc une forte valorisation de l'Auteur.

Dans un deuxième temps, l'Auteur tire tout simplement sa légitimité du fait que c'est Dame Musique en personne qui lui a confié la mission d'écrire cette Déploration. Pourtant, Guillaume Crétin dit qu'elle lui a

42. E. R. CuRTIUs, La Littérature européenne et le Moyen Âge latin, trad. J. BréJoux, Paris, 1956, p. 103

43. Ibid p. 196 
ordonné d'écrire «tout ce que j'avoye veu» (v. 230), et non entendu: il n'est pas censé rendre la musique, mais les événements du songe. Or, il s'est essayé à en transmettre la musique. Il écrit ainsi :

Pour hault louer le melodieux son,

La voix, le chant et subtile façon

De ce vaillant renommé Tresorier (v. 271-273)

Son poème dépasse donc le cadre de la besogne qui lui a été confiée. Ainsi, et par le mécanisme que j'ai décrit ci-dessus, l'injonction de Dame Musique, légitimante en soi, devient le prétexte à l'auto-légitimation poétique d'un Auteur zélé qui a fait plus qu'on ne lui demandait ${ }^{44}$.

Ensuite, les rondeaux chantés pendant le songe peuvent être assimilés à des déplorations en miniature ${ }^{45}$. Cela renforce encore le caractère d'autolégitimation du poème puisque Guillaume Crétin, par le truchement de cette mise en abyme, se place sur le même pied - celui du poète laudateur que des personnages aussi grandioses qu'Orphée, David, Tubal et Chiron. On comprend dès lors la signification profonde de l'insertion lyrique: elle doit permettre la mise en abyme et, par là même, l'admission du je de la narration au rang de ceux qui ont porté la parole lyrique dans le poème. La non-coïncidence préalable des je lyriques et du je de l'Auteur est nécessaire à l'assimilation légitimante. Par le truchement de l'insertion lyrique, le narrateur se rend ainsi lui-même capable de lyrisme - et ce d'autant plus que la non-coïncidence est une fiction puisque les rondeaux sont de Crétin : les lecteurs, qui ne l'ignorent évidemment pas, entrent ainsi en connivence avec le poète aux dépens de Tubal, David, Orphée et Chiron.

Enfin, dans la conclusion de son poème, l'Auteur appelle ses contemporains à pleurer et à chanter en mémoire d'Ockeghem:

44. À ce propos, il est intéressant de comparer la situation de Guillaume Crétin avec celle de Jean Molinet, au sujet duquel l'Auteur note: «Et veu aussi que Dame Rethorique En tous voz faictz vous porte et favorise» (v. 290-291). Molinet, directement soutenu par la prosopopée de l'art qu'il pratique, semble ainsi mieux légitimé que Crétin, qui est justifié par l'intervention d'une Dame qui n'est pas la sienne, de façon indirecte, différée et presque métalittéraire: c'est parce que le lecteur est en train de lire ce poème finalement écrit que son auteur s'en trouve légit 列

45. D'ailleurs, aussi artificiel que ce stratagème puisse paraître, je remarque que, si l'on met à la suite les refrains des quatre rondeaux, on obtient la séquence suivante: «C'est Okergan. En chant de pleur. Musiciens. Plorer le fault», qui n'est rien d'autre qu'une synthèse schématique mais exacte de la Déploration. En effet, Guillaume Crétin a d'abord, par le récit du songe, rapporté le génie d'Ockeghem, avant d'appeler ses contemporains musiciens à chanter et pleurer le grand homme disparu. La mise en abyme est totale, en plus que d'être espiègle.
Seigneurs de Tours et peuple regrettez

Celluy qu'on doibt plus plaindre que ne dys (v. 381-382)

Chantres, plorez ce notable Seigneur,

En visitant ses doulx chantz angelicques (v. 389-390)

La Déploration revêt donc une dimension performative, puisqu'elle prétend redoubler le monde du songe allégorique - où des personnages mythiques et légendaires, liés à la musique, et d'illustres compositeurs pleuraient et chantaient pour Ockeghem - dans l'épaisseur du monde réel. Ainsi la parole, présentée comme impuissante à dire (donc à dire la musique) - ce qui justifiait l'idée d'une supériorité de la musique sur la poésie -, se révèle pour finir suffisamment puissante pour faire la musique, pour l'informer, au sens où, par l'injonction du chant sobre, elle lui donne forme, afin de la tirer du piège dans lequel elle s'était enfermée: le paradoxe du chantplainte. La parole devient donc performative, et la poésie prend le relais là où la musique est impuissante parce que confrontée à une aporie. La poésie marque dès lors sa supériorité sur la musique en désamorçant l'opposition paradoxale de l'harmonie des chants et de la disharmonie des cœurs.

En définitive, cette Déploration, qui semblait à première vue célébrer la musique, présente toutes les caractéristiques d'une auto-légitimation poétique: l'Auteur y procède en effet non seulement à la valorisation de la poésie fraîchement affranchie de la musique, mais aussi à celle du poète, en tant que technicien du mot, musicien du verbe, selon l'idéal de raffinement et de préciosité stylistiques propre aux Grands Rhétoriqueurs ${ }^{46}$. Ainsi, Guillaume Crétin inscrit son texte dans une tradition bien établie, voire formulaire, qu'il réinvente en la détournant: en célébrant poétiquement la mort d'un musicien - à la différence d'un Robertet écrivant sur Chastelain Crétin peut faire disjoncter le traditionnel topos d'humilité. D'autre part parce qu'ils sont à la fois le reflet des angoisses d'un siècle - le $\mathrm{XV}^{\mathrm{e}}-$ particulièrement douloureux et la réponse à ces angoisses, l'éloge funèbre et plus largement la poésie de circonstance entendent, écrit Claude Thiry, «affirmer bien haut la grandeur de l'homme et de ses actions face au temps, à l'oubli, à la mort ${ }^{47}$ ». Or, selon la logique propre à l'éloge funèbre, la solennité conférée à la parole du poète par le récit du songe allégorique

46. N'est-ce pas précisément afin de célébrer cette qualité de musicien que Guillaume Crétin se met en scène, en tant qu'Auteur, sous la protection de Dame Musique plutôt que sous celle de Dame Rhétorique? La distinction entre Jean Molinet et Guillaume Crétin, respectivement légitimés par Dame Rhétorique et par Dame Musique (voir note ci-dessus) $\mathrm{s}$ 'inscrit donc bien dans l'hypothèse de l'auto-légitimation.

47. C. ThiRY, «La poésie de circonstance», dans Grundriss der Romanischen Litteraturen..., vol. viII/1, D. PoIRION (dir.), La Littérature française aux XIV et $X V^{e}$ siècles. p. 138. 
aurait dû être mise au service de la glorification du compositeur défunt. Mais Guillaume Crétin se joue des attentes de son lecteur et prononce in fine l'éloge de la poésie au moins autant que celui d'Ockeghem. Cela n'est naturellement pas anodin. Dans un temps où, alors que la vie de cour se pare d'une intensité et d'un formalisme inédits, la complainte - expurgée de ses atours intimistes - prononcée à la mort d'un prince connaît une fortune importante, comme le souligne Robert Deschaux ${ }^{48}$, le fait de prononcer l'éloge d'un musicien est un acte littéraire significatif: «Pour l'auteur de complainte, l'artiste tend ainsi à devenir l'égal du prince, à l'heure de la mort ${ }^{49}$.» En couronnant Ockeghem, et parce que, ce faisant, il se couronne lui-même, Guillaume Crétin ne fait donc rien d'autre que de proclamer cette orgueilleuse sentence: il est le prince des poètes... ou, à tout le moins, un prince parmi les poètes.

Thibaut RADOMME - Université catholique de Louvain, Institut des Civilisations, Arts et Lettres (INCAL)

\section{Guillaume Crétin et la Déploration sur le trépas de Jean Ockeghem : les} chœurs, les cœurs et la poésie

Cet article propose une étude du poème Déploration sur le trépas de Jean Ockeghem, écrit par Guillaume Crétin à l'extrême fin du XVe siècle selon deux axes de lecture principaux: la représentation de l'harmonie des chours et de la disharmonie des coeurs d'une part, et la problématisation du topos d'humilité de l'auteur, d'autre part. Après un la prove introduction du topos dhu la diste de l'auteur, d'autre part. Apres une breve introduction qu'à une perspective gén con sur le poème, la première partie de l'article qu'a une perspective generale sur le poème, la premiere partie de l'article (harte du premier axe de lecture. il y est montre que, si la beaute des chants (harmonie des chœurs) semble a priori contraster avec le trouble des sentiments (disharmonie des cœurs), les frontières sont en fait brouillees et les chœurs sont gagnés par le desordre des cœurs, traduisant le rejet de la gaieté qui caractérise le chant funébre. La seconde partie de l'article entend questionner la position d'infériorité dans laquelle se place Guillaume Crétin en tant qu'auteur: après s'être affirmé incapable d'écrire le poème qu'on lui enjoint de composer, après en avoir appelé à ses illustres prédécesseurs et contemporains et avoir reconnu la supériorité de la musique sur la poésie, lauteur semble en effet prendre à revers les attentes du lecteur et, par un habile jeu de mise en abyme et une exploitation de la valeur performative du poème, se couronner comme le prince des poètes.

harmonie - mise en abyme - topos d'humilité - complainte - poésie de circonstance
Guillaume Crétin and the Déploration sur le trépas de Jean Ockeghem : Choruses, Hearts and Poetry

This article offers a study of the poem Déploration sur le trépas de Jean Ockeghem, written by Guillaume Crétin at the extreme end of the fifteenth century, in a double perspective: the representation of choruses'harmony and hearts'disharmony on one hand, and the problematisation of the topos of author's humility on the other hand. After a brief introduction, in which I give a contextual background about the concepts of harmony and disharmony as well as a general overview of the poem, the first part of the article deals with the first perspective of reading: if choruses'harmony seems at first sight to contrast with hearts'disharmony, borders are in fact blurred and choruse are contaminated by the disorder of feelings, which results in the rejection of happiness that characterizes funeral singing. In the second part of the article, I want to examine the position of inferiority in which Guillaume Crétin puts himself as an author. Indeed, whilst he claims to be unable to write the poem that he has been asked to compose and calls for the help of his illustrious predecessors and famous contemporaries, he recognizes the superiority of music over poetry. However, the author seems to deceive the expectations of the reader and, through an ingenious mise en abyme and by exploiting the performative dimension of the poem, to crown himself as the prince of the poets.

harmony - mise en abyme - topos of humility - complaint - occasional poetry

48. R. Deschaux, «Le Lai et la Complainte...», p. 82.

49. Ibid., p. 83. 